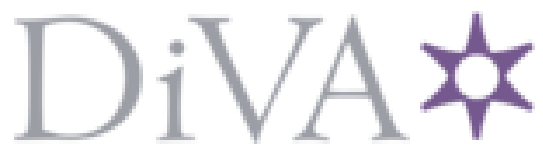

http://www.diva-portal.org

This is the published version of a paper published in .

Citation for the original published paper (version of record):

Bittencourt, A C., Saarinen, K., Sander Tavallaey, S., Gunnarsson, S., Norrlöf, M. (2014) A data-driven approach to diagnostics of repetitive processes in the distribution domain - Applications to gearbox diagnostics in industrial robots and rotating machines

Mechatronics, 24(8): 1032-1041

Access to the published version may require subscription.

N.B. When citing this work, cite the original published paper.

Permanent link to this version:

http://urn.kb.se/resolve?urn=urn:nbn:se:kth:diva-298574 


\title{
A data-driven approach to diagnostics of repetitive processes in the distribution domain - Applications to gearbox diagnostics in industrial robots and rotating machines
}

\author{
André Carvalho Bittencourt ${ }^{\mathrm{a}, *}$, Kari Saarinen ${ }^{\mathrm{b}}$, Shiva Sander-Tavallaey ${ }^{\mathrm{b}}$, Svante Gunnarsson ${ }^{\mathrm{a}}$, \\ Mikael Norrlöf ${ }^{\mathrm{a}, \mathrm{c}}$ \\ ${ }^{a}$ Department of Electrical Engineering, Linköpings University, Linköping, Sweden \\ ${ }^{\mathrm{b}}$ ABB Corporate Research, Västerås, Sweden \\ ${ }^{\mathrm{c}}$ ABB Robotics, Västerås, Sweden
}

\section{A R T I C L E I N F O}

\section{Article history:}

Received 1 February 2013

Accepted 27 January 2014

Available online 28 February 2014

\section{Keywords:}

Repetitive processes

Diagnostics

Fault detection and isolation

Wear

Vibration analysis

Gearbox

\begin{abstract}
A B S T R A C T
This paper presents a data-driven approach to diagnostics of systems that operate in a repetitive manner. Considering that data batches collected from a repetitive operation will be similar unless in the presence of an abnormality, a condition change is inferred by comparing the monitored data against an available nominal batch. The method proposed considers the comparison of data in the distribution domain, which reveals information of the data amplitude. This is achieved with the use of kernel density estimates and the Kullback-Leibler distance. To decrease sensitivity to disturbances while increasing sensitivity to faults, the use of a weighting vector is suggested which is chosen based on a labeled dataset. The framework is simple to implement and can be used without process interruption, in a batch manner. The approach is demonstrated with successful experimental and simulation applications to wear diagnostics in an industrial robot gearbox and for diagnostics of gear faults in a rotating machine.
\end{abstract}

(c) 2014 Elsevier Ltd. All rights reserved.

\section{Introduction}

In the manufacturing industry, preventive scheduled maintenance is a common approach used to improve equipment's safety, reliability, availability and maintainability. This setup delivers high availability, reducing operational costs (e.g. small downtimes) with the drawback of high maintenance costs since unnecessary maintenance actions might take place. Condition based maintenance (CBM), "maintenance when required", can deliver a good compromise between maintenance and operational costs, reducing the overall cost of maintenance. The extra challenge of CBM is to define methods to determine the condition of the equipment. This can be done by comparing the observed and expected (known) behaviors of the system through an algorithm. The output of such algorithm

\footnotetext{
* Corresponding author. Tel.: +4613282636.

E-mail addresses: andrecb@isy.liu.se (A.C. Bittencourt), kari.saarinen@se.abb. com (K. Saarinen), shiva.sander-tavallaey@se.abb.com (S. Sander-Tavallaey), svante@isy.liu.se (S. Gunnarsson), mikael.norrlof@se.abb.com (M. Norrlöf).

URL: http://www.control.isy.liu.se/andrecb (A.C. Bittencourt).
}

is a quantity sensitive to a fault, ${ }^{1}$ i.e. a fault indicator, which can be monitored to determine the current state of the system (e.g. healthy/ broken).

A common approach to generate fault indicators is based on the use of residuals, i.e. fault indicators that are achieved based on deviations between measurements and the output of a system model, see e.g. [25,27]. A system model is a map from input to output data. A system model provides important information about the behavior of the system, facilitating the generation of fault indicators. Different approaches for residual generation are based on, e.g., observers, parity-space and parameter identification. When a model of the system is not available or it is too costly to be developed, alternatives are still possible. These alternatives will typically require extra (redundant) sensory information or expert knowledge about the measured data, e.g., their nominal frequency content or the use of labeled data. Essentially, however, any method will attempt to generate quantities that can be used to infer the actual condition of the system given the available knowledge and observations, i.e. data.

\footnotetext{
${ }^{1}$ A fault is defined as a deviation of at least one characteristic property of the system from the acceptable/usual/nominal condition.
} 
The use of model-based approaches is common for diagnostics of machines. For robotics, many approaches have been suggested based on the use of nonlinear observers, where the observer stability is typically guaranteed by analyses of the decay rate of a candidate Lyapunov function, see e.g. $[9,10,12,15,20,22,30]$. Observers can also be designed based only on data, without a description of the system based on first principles. Data-driven design of observers are typically based on subspace identification of linear models and have been suggested for fault detection in $[14,16,42,44]$. Parameter estimation is also a natural approach to model-based diagnostics because of the physical interpretation of the system parameters, see e.g. [5,21,29].

In cases where the data are ordered with time, signal-driven methods are common for machinery diagnostics. These are typically based on the use of integral transforms, e.g. Fourier, Radon, Karhunen-Loéve or Wavelet. Each transform will enhance different properties in the transformed domain and are suitable depending on the characteristics of the signal, e.g. periodic, stationary, etc. The analysis of data in the frequency domain or time-frequency has found particular success in the monitoring of rotating machines, see e.g. $[13,19,23-25,38,40]$. Some approaches have also been proposed for the diagnostics of industrial robots with the use of additional sensory information $[18,32]$.

A common challenge to data-driven methods is that the data characteristics' will vary depending on the operating points, which may complicate determination of fault presence. This is particularly restricting for an industrial robot where the kinematic configuration of the robot may give varying load torques at the joints during motion. This shortcoming can be circumvented by considering data from a specific operation of the system, e.g. under repetition. A repetitive operation is found in various applications, e.g. in automated manufacturing. Repetition can also be forced with the execution of specific diagnostic routines but with the drawback of reduced availability. Much attention has been given recently to repetitive processes $[36,37]$. Study of repetitive processes have mainly focused on control $[35,39]$ and estimation problems $[1,2]$. A few approaches have been also suggested for model-based diagnostics, e.g. [43].

In this paper, a data-driven method is proposed for the generation of fault indicators for systems that operate in a repetitive manner. It is considered that in case the condition of the system is nominal, data batches collected from repetitive executions of the system will be similar to each other and will differ if the condition changes. The comparison of a given data batch against a nominal one can thus be used to infer whether an abnormality is present. The fault indicator proposed here relates to changes in the distribution of these batches of data. This is made possible with the use of kernel density estimators and the Kullback-Leibler distance between distributions. A distribution domain approach does not consider the dynamics of the system generating the data as is the case in, e.g., observer-based approaches. As it will be presented, this leads to very simple diagnostics solutions that can perform well in practical setups.

The proposed framework was initially developed with the interest focused on the diagnostics of wear in industrial robots and a preliminary version of the work can be found in [7]. Here, more aspects are covered, including approaches to detection, isolation and reduction of sensitivity to disturbances. Also, more experimental and simulation results are presented for the robotics application. An additional application is also included for diagnostics of rotating machinery based on vibration data collected from an accelerometer. The paper is organized as follows; a general presentation of data-driven diagnostics and repetitive systems is given in Section 2, followed by the presentation of the proposed approach for diagnostics in the distribution domain in Section 3. The applications are presented in Sections 4 and 5. Conclusions and future work are given in Section 6.

\section{Data-driven diagnostics and repetitive systems}

Consider a general system from which it is possible to extract a sequence of data batches,

$\mathbf{Y}_{K}=\left[\mathbf{y}_{1}, \cdots, \mathbf{y}_{k}, \cdots, \mathbf{y}_{K}\right]$

where $\mathbf{y}_{k}=\left[y_{k, 1}, \cdots, y_{k, n}, \cdots, y_{k, N}\right]^{T}$ denotes the data vector in $\mathbb{R}^{N}$ (e.g. measurements or known inputs) with batch index $k$ and element index $n$. The sequence $\mathbf{y}_{k}$ could have been generated as the result of deterministic and stochastic inputs, $\mathbf{Z}_{K}$ and $\mathbf{V}_{K}$, where $\mathbf{V}_{K}$ is unknown, and $\mathbf{Z}_{K}$ may have known and unknown components. For example, the data generation mechanism could be modeled as

$\mathbf{y}_{k}=h\left(\mathbf{z}_{k}, \mathbf{v}_{k}\right)$,

where $h(\cdot)$ is an unknown function. Let the set of deterministic inputs $\mathbf{Z}_{K}$ be categorized in three distinct groups, $\mathbf{R}_{K}, \mathbf{D}_{K}$ and $\mathbf{F}_{K}$. The sequence $\mathbf{f}_{k}$ is unknown and of interest (a fault), $\mathbf{r}_{k}$ is known (e.g. references or control inputs) and $\mathbf{d}_{k}$ is unknown (disturbances).

The objective is to define a data-driven framework for the generation of fault indicators to determine the presence of a fault $\mathbf{f}_{k}$. Because a data-driven approach depends on availability of data, it is assumed that data generated under no fault is available. Let $\mathcal{Y}^{0}=\left\{\mathbf{y}_{k}: \mathbf{f}_{k}=\mathbf{0}\right\}$ denote the set of data batches that were generated under no fault, the following assumption is made:

\section{A-1 (Nominal data are available) A sequence $\mathbf{y}^{0} \in \mathcal{Y}^{0}$ is available.}

The rationale is then to generate fault indicators from the comparison of the nominal data $\mathbf{y}^{0}$ (available from Assumption A-1) against the remaining sequences $\mathbf{y}_{k}$. In order to generate fault indicators for $\mathbf{y}_{k}$ using the nominal data $\mathbf{y}^{0}$, two basic questions arise:

\section{Q-1 How to characterize a sequence $\mathbf{y}_{k}$ ?}

Q-2 How to compare the sequences $\mathbf{y}^{0}, \mathbf{y}_{k}$ ?

The first question targets the issue of finding a data processing mechanism of $\mathbf{y}_{k}$, written in a general form as $g_{k} \triangleq g\left(\mathbf{y}_{k}\right): \mathbb{R}^{N} \mapsto \mathbb{G}$ with domain $\mathbb{G}$, whose output enhances the ability to discriminate the presence of non-zero $\mathbf{f}_{k}$. Given the nominal data in the transformed domain $g^{0} \triangleq g\left(\mathbf{y}^{0}\right)$, fault indicators can be achieved from the comparison between $g^{0}$ and $g_{k}$. This is typically, but not necessarily, done with the use of a distance function represented as $d\left(g^{0}, g_{k}\right): \mathbb{G} \times \mathbb{G} \mapsto \mathbb{R}_{0}^{+}$. Different distances are possible depending on the domain $\mathbb{G}$. For example, for diagnostics of rotating machines $g_{k}$ could be the spectra of $\mathbf{y}_{k}$ and $d(\cdot, \cdot)$ a spectral distance, see e.g. [3].

\subsection{Detection, performance and isolation}

Let $\mathcal{D}^{m, n}=\left\{d\left(g_{i}, g_{j}\right): \mathbf{y}_{i} \in \mathcal{Y}^{m}, \mathbf{y}_{j} \in \mathcal{Y}^{n}\right\}$, then $\mathcal{D}^{0,0}$ describes the behavior of the fault indicator when no fault is present and $\mathcal{D}^{0 . f}$, where $\mathcal{Y}^{f}=\left\{\mathbf{y}_{k}: \mathbf{f}_{k} \neq \mathbf{0}\right\}$, describes all possible faulty behaviors. A criterion for detectability of an abnormality is that $\mathcal{D}^{0 . f}$ is not completely contained in $\mathcal{D}^{0,0}$, i.e. $\mathcal{D}^{0, f} \backslash \mathcal{D}^{0,0} \neq \emptyset$. Since the distance $d\left(g^{0}, g_{k}\right)$ measures how far $g_{k}$ is from the nominal $g^{0}$, it is expected that it will remain close to zero if $d\left(g^{0}, g_{k}\right) \in \mathcal{D}^{0,0}$ and to deviate to positive values if $d\left(g^{0}, g_{k}\right) \in \mathcal{D}^{0 . f}$. Suppose that it is possible to find a threshold $\hbar$ such that $d\left(g^{0}, g_{k}\right) \leqslant \hbar$ most of the times when $d\left(g^{0}, g_{k}\right) \in \mathcal{D}^{0,0}$, a simple criterion for detection is then to consider a threshold check. Let $\mathcal{H}^{0}$ denote the hypothesis that no fault is 
present, i.e. $\mathbf{y}_{k} \in \mathcal{Y}^{0}$, and $\mathcal{H}^{1}$ denote the alternative hypothesis that a fault is present, i.e. $\mathbf{y}_{k} \in \mathcal{Y}^{f}$, then the decision mechanism is

$d\left(g^{0}, g_{k}\right) \stackrel{\mathcal{H}^{1}}{\gtrless} \hbar$

and reads, decide for $\mathcal{H}^{1}$ if $d\left(g^{0}, g_{k}\right) \geqslant \hbar$ otherwise decide for $\mathcal{H}^{0}$. The probabilities of error for this decision rule can be quantified given the probability distribution of the fault indicator under the different hypotheses, denoted $p\left(d \mid \mathcal{H}^{0}\right)$ and $p\left(d \mid \mathcal{H}^{1}\right)$. The probability of a false detection $P_{f}$, i.e. deciding for a fault when none is present, and of correct detection $P_{d}$, i.e. deciding for a fault when it is present can be evaluated by

$P_{f}=\int_{h}^{\infty} p\left(x ; d \mid \mathcal{H}^{0}\right) \mathrm{d} x, \quad P_{d}=\int_{h}^{\infty} p\left(x ; d \mid \mathcal{H}^{1}\right) \mathrm{d} x$.

Notice that for a fixed $P_{f}$ there is an associated $\hbar$ (this is known as the Neyman-Pearson criterion for threshold selection [41]) and therefore a $P_{d}$. For a satisfactory performance of the fault indicator, low $P_{f}$ and high $P_{d}$ are typically desirable.

Since this is a data-driven framework, data from the different fault types are needed a priori to address the isolation problem, i.e. determination of the fault type present. Let $\mathcal{Y}^{m}=\left\{\mathbf{y}_{k}: \mathbf{f}_{k}=\mathbf{f}^{m}\right\}$ denote the set of data generated under the $m$ th fault type with $M$ possible fault modes, i.e. $m \in \mathcal{M}=[1,2, \cdots, M]$. For fault isolation it is assumed:

\section{A-2 (Data from each fault type are available) A sequence $\mathbf{y}^{m} \in \mathcal{Y}^{m}$ is available for each $m$ th fault type.}

Once a fault is detected, the fault type can be determined by choosing $\mathbf{y}^{m}$ that is closest to $\mathbf{y}_{k}$ in the sense of the transformation $g(\cdot)$ and distance $d(\cdot, \cdot)$. That is, decide for presence of the $m^{*}$ fault type according to

$m^{*}=\arg \min _{m \in \mathcal{M}}\left(g^{m}, g_{k}\right)$.

\subsection{Repetitive systems}

The amount of overlaps between the sets $\mathcal{D}^{0,0}$ and $\mathcal{D}^{0 . f}$ relates to how difficult it will be to determine the presence of a fault. Ultimately, no overlap is present, i.e. $\left|\mathcal{D}^{0,0} \cap \mathcal{D}^{0, f}\right|=0$, when it is possible to determine the presence of a fault with no errors. This is particularly difficult to achieve since $\mathbf{y}_{k}$, and thus $\mathcal{D}^{0,0}$ and $\mathcal{D}^{0 . f}$, are affected by other inputs than faults. Because $\left|\mathcal{D}^{0,0} \cap \mathcal{D}^{0 . f}\right| \leqslant \min \left|\mathcal{D}^{0,0}\right|,\left|\mathcal{D}^{0, f}\right|$, an attempt to try to reduce the overlaps is to reduce the size of the sets themselves. This can be achieved by restricting the behavior of the data $\mathbf{y}_{k}$ or, conversely, by restricting the possible input space. This paper focuses on monitoring data collected from a repetitive operation of the system. A repetitive operation is meant by a system that executes the same trajectory $\mathbf{r}$ over each data batch collected. This type of data is represented by the set

$\mathcal{Y}_{r}=\left\{\mathbf{y}_{k}: \mathbf{r}_{k}=\mathbf{r}\right\}$.

Monitoring data $\mathbf{y}_{k} \in \mathcal{Y}_{r}$ simplifies the problem since the behavior of the data will be more predictable. Examples of systems that behave repetitively are common in automation applications. A repetitive operation can also be forced in case $\mathbf{r}$ can be chosen freely; for instance data can be collected based on the execution of scheduled diagnostics tests. This setup is also commonly found in vibration analyses and in signal-based diagnostics, where data are collected under particular operating conditions, e.g. of speed, load and acceleration.

Considering that faults are detectable for a system operating under repetition, there may still be overlaps between the sets
$\mathcal{D}_{r}^{0,0}$ and $\mathcal{D}_{r}^{0, f}$ defined by $\mathcal{Y}_{r}^{0}=\mathcal{Y}^{0} \cap \mathcal{Y}_{r}$ and $\mathcal{Y}_{r}^{f}=\mathcal{Y}^{f} \cap \mathcal{Y}_{r}$. This is due to the effects of disturbances $\mathbf{d}_{k}$ and noise $\mathbf{v}_{k}$. By collecting data in a controllable manner, it might be possible to ensure repetition of the disturbance term, i.e. to have $\mathbf{d}_{k}=\mathbf{d}$ for all batches. This will be however too restrictive for most applications. Even if $\mathbf{d}_{k}=\mathbf{d}$ is possible, the noise components will always affect the data. To broaden the scope of the framework, a clear determination of the fault presence despite $\mathbf{d}_{k}$ and $\mathbf{v}_{k}$ is desirable, leading to the question:

\section{Q-3 How to handle non-repetitive disturbances $\mathbf{d}_{k}$ and noise $\mathbf{v}_{k}$ ?}

Questions Q-1-Q-3 are addressed in the next section which defines the suggested approach for diagnostics of repetitive systems in the distribution domain.

\section{A distribution domain approach}

\subsection{Characterizing the data - kernel density estimate}

The alternative pursued in this work is to consider the distribution of $\mathbf{y}_{k}$, which contains information about the amplitude behavior of the data. Even though information contained in the ordering may be lost, this is a valid approach since the effects of a fault often appear as changes in amplitude. Since data batches from a repetitive operation are considered, i.e. $\mathbf{y}_{k} \in \mathcal{Y}_{r}$, it is expected that the data distribution will remain similar in case no fault is present. Because the mechanisms that generated the data are considered unknown, the use of a nonparametric estimate of the distribution of $\mathbf{y}_{k}$ is a suitable alternative. A nonparametric estimate of the distribution $p(y)$ based on the data vector $\mathbf{y}$ can be achieved from the empirical characteristic function. For a scalar random variable with probability density function $p(y)$, the characteristic function $\vartheta: \mathbb{R} \rightarrow \mathbb{C}$ with argument $v$ is defined as, [17]:

$\vartheta(v)=\mathbb{E}\left[e^{i v y}\right]=\int_{-\infty}^{\infty} e^{i v y} p(y) \mathrm{d} y=\mathbf{F}^{-1}\{p(y)\} 2 \pi$,

where $\mathbf{F}^{-1}\{\cdot\}$ is the inverse Fourier transform. So the density function can be found from the characteristic function through its Fourier transform. Given the sample $\mathbf{y}=\left[y_{1}, \cdots, y_{N}\right]$, the empirical estimate of $\vartheta(v)$ is given by

$\hat{\vartheta}(v)=\frac{1}{N} \sum_{n=1}^{N} e^{i v y_{n}}$,

the objective is then to estimate the density function from $\hat{\vartheta}(v)$. This is essentially a spectrum estimation problem. A direct estimation of the density function from the Fourier transform of $\hat{\vartheta}(v)$ will however lead to an estimate with increased variance for large values of $v$, [28]. To avoid this problem, the empirical estimate of the characteristic function is multiplied with a weighting function $\psi_{h}(v)=\psi(h v)$. The weighting function is typically symmetric, satisfying $\psi(0)=1$ and tends to zero when $v$ tends to infinity. The density estimate is then given by

$$
\begin{aligned}
\hat{p}(y) & =\frac{1}{2 \pi} \mathbf{F}\{\hat{\vartheta}(v) \psi(h v)\}=\frac{1}{2 \pi} \int_{-\infty}^{\infty} \frac{1}{N} \sum_{n=1}^{N} e^{i v\left(y_{n}-y\right)} \psi(h v) \mathrm{d} v \\
& =\frac{1}{N h} \sum_{n=1}^{N} \kappa\left(\frac{y_{n}-y}{h}\right)=\frac{1}{N} \sum_{n=1}^{N} \kappa_{h}\left(y-y_{n}\right),
\end{aligned}
$$

where $\kappa_{h}(y) h=\mathbf{F}^{-1}\left\{\psi_{h}(v)\right\}$. The function $\kappa_{h}(y)$ is a kernel function, satisfying $\kappa_{h}(\cdot) \geqslant 0$ and that integrates to 1 . The resulting estimate is known as a kernel density estimate (KDE) and can also be generalized to the multidimensional case [11]. The bandwidth parameter $h$ controls the smoothness of the resulting estimate, increasing the smoothness for larger values of $h$. When $h \rightarrow 0$, the kernel function 
approaches a Dirac delta and the resulting estimate will be a set of impulses located at the data points. Typical kernel functions and their Fourier transforms are shown in Fig. 1. In this work, a Gaussian kernel is used with $h$ optimized for Gaussian distributions, see [8]. For a detailed treatment of kernel density estimators and criteria/ methods for choosing $h$ see $[8,11,26,33]$.

\subsection{Comparing sequences - Kullback-Leibler distance}

In statistics and information theory, the Kullback-Leibler divergence (KLD) is commonly used as a measure of difference between two probability distributions. For two continuous distributions on $y, p(y)$ and $q(y)$, it is defined as, [34]

$D_{\mathrm{KL}}(p \| q) \triangleq-\int_{-\infty}^{\infty} p(y) \log \frac{q(y)}{p(y)} \mathrm{d} y$.

The KLD satisfies $D_{\mathrm{KL}}(p \| q) \geqslant 0$ (Gibbs inequality), with equality if and only if $p(y)=q(y)$. The KLD is in general not symmetric, $D_{\mathrm{KL}}(p \| q) \neq D_{\mathrm{KL}}(q \| p)$. The quantity

$\mathrm{KL}(p \| q) \triangleq D_{\mathrm{KL}}(p \| q)+D_{\mathrm{KL}}(q \| p)$,

known as the Kullback-Leibler distance, is however symmetric. An answer to Question Q-2 can therefore be given with the use of the KL distance defined in (11). From Assumption A-1, fault-free data are always available, so that $\mathbf{y}^{0}$ is known and $\hat{p}^{0}(y)$ can be computed. The quantities $\operatorname{KL}\left(\hat{p}^{0} \| \hat{p}_{k}\right)$ can therefore be used as a fault indicator, remaining close to 0 in case $\hat{p}^{0}(y)$ is close to $\hat{p}_{k}(y)$ and otherwise deviating to positive values.

\subsection{Handling non-repetitive disturbances and noise - data weighting}

One way to address Question Q-3 is to weight the raw data $\mathbf{y}_{k}$ according to prior knowledge of the effects of faults, disturbances and noise in order to give more relevance to parts of the data that relate to a fault. The approach considered here will assume availability of a labeled dataset, where the fault status (present or not) is known to each component $\mathbf{y}_{k}$ and is the same to each of its elements $y_{k, n}$. The disturbance vector $\mathbf{d}_{k}$ and noise $\mathbf{v}_{k}$ should contain variations that are expected to be found during the system's operation.

The dataset $\mathbf{Y}_{K}=\left[\mathbf{Y}_{K_{0}}^{0}, \mathbf{Y}_{K_{f}}^{f}\right]$ contains $K_{0}$ fault-free data, $\mathbf{y}^{0} \in \mathcal{Y}_{r}^{0}$, and $K_{f}=K-K_{0}$ faulty data $\mathbf{y}^{f} \in \mathcal{Y}_{r}^{f}$. Each batch $\mathbf{y}_{k}$ is weighted according to

$\overline{\mathbf{y}}_{k} \triangleq \mathbf{w} \circ \mathbf{y}_{k}$,

where $\circ$ denotes the Hadamard product (element-wise multiplication). This yields the weighted dataset

$\overline{\mathbf{Y}}_{K} \triangleq\left[\overline{\mathbf{Y}}_{K_{0}}^{0}, \overline{\mathbf{Y}}_{K_{f}}^{f}\right]=\left[\overline{\mathbf{y}}_{1}^{0}, \cdots, \overline{\mathbf{y}}_{K_{0}}^{0}, \overline{\mathbf{y}}_{1}^{f}, \cdots, \overline{\mathbf{y}}_{K_{f}}^{f}\right]$.

The objective is to choose $\mathbf{w}$ to maximize the sensitivity to faults while decreasing sensitivity to disturbances and noise. Considering the basic framework presented in Sections 3.1 and 3.2, a natural criterion would be to choose $\mathbf{w}$ according to its effects to $\operatorname{KL}\left(\hat{\bar{p}}_{i} \| \hat{\bar{p}}_{j}\right)$, where $\hat{\bar{p}}_{i}$ is the $\mathrm{KDE}$ of $\overline{\mathbf{y}}_{i}$ and therefore dependent on $\mathbf{w}$. When $\mathbf{y}_{i} \in \mathcal{Y}_{r}^{0}$ and $\mathbf{y}_{j} \in \mathcal{Y}_{r}^{f}$, the distance should be maximized and if $\mathbf{y}_{i}, \mathbf{y}_{j} \in \mathcal{Y}_{r}^{0}$, it should be minimized. A general solution to this problem is however difficult since it depends on how $\hat{\bar{p}}_{k}(y)$ is computed (e.g. the kernel function chosen) and optimization over (11).

In this work, simpler criteria are considered in a compromise to explicit solutions. As it will be shown, the results are related to linear discriminant analysis (LDA) used in classification problems, see e.g. [4]. In LDA, the inner product $\mathbf{w}^{T} \mathbf{y}$ is used instead of the Hadamard product. While the data are reduced to a scalar quantity in LDA, the use of the Hadamard product keeps the data dimensionality and therefore the KDE can still be computed, yielding the estimates $\hat{\bar{p}}_{k}$. Furthermore, the objective in LDA is to obtain a classifier. Here, in contrast, $\mathbf{w}$ is chosen as to achieve average separation between faulty and fault-free data while giving small variability to disturbances and noise.

Notice that once the weights are chosen, the same vector $\mathbf{w}$ is used for new data batches. For consistency, it is thus required that the data sequences are synchronized. This can however be overcome in case the weights are strongly correlated to measured data.
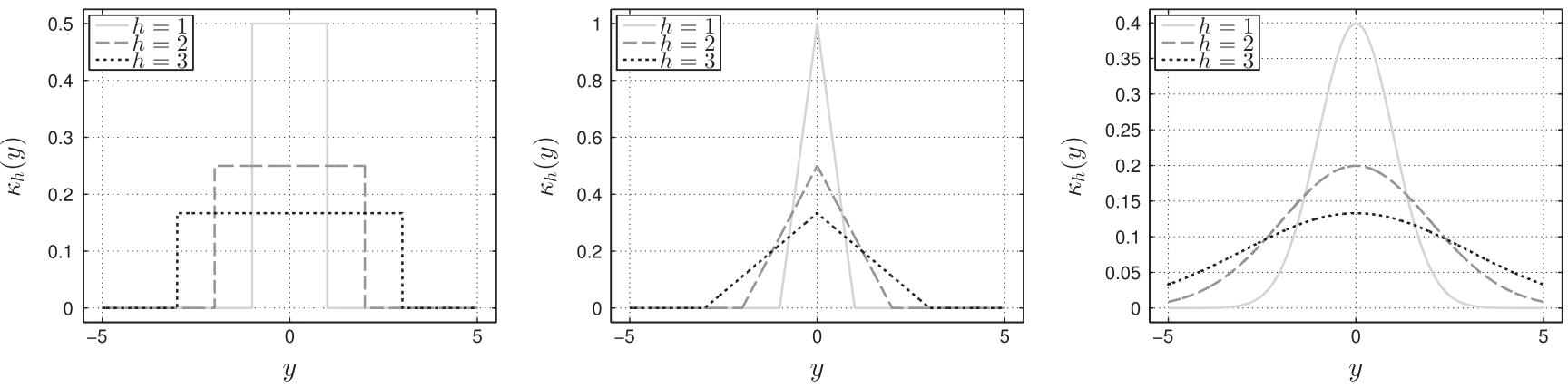

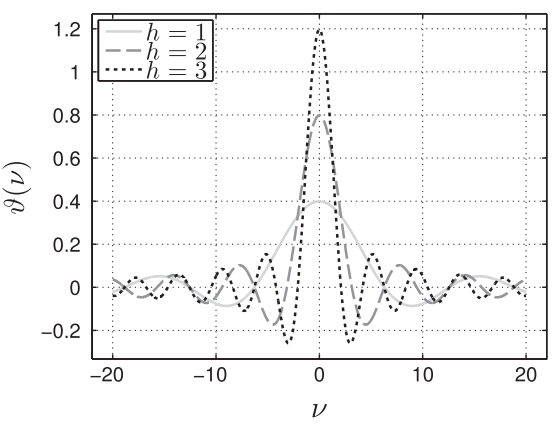

Uniform

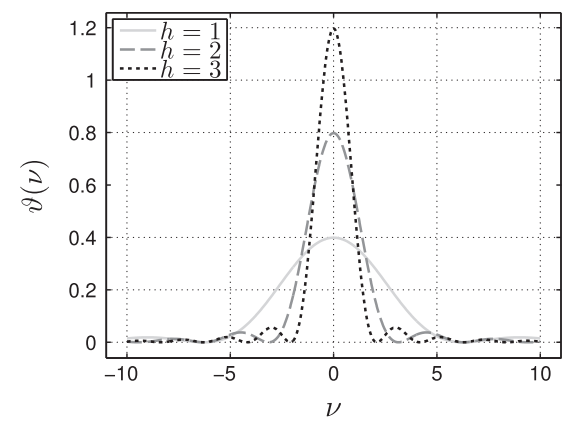

Triangular

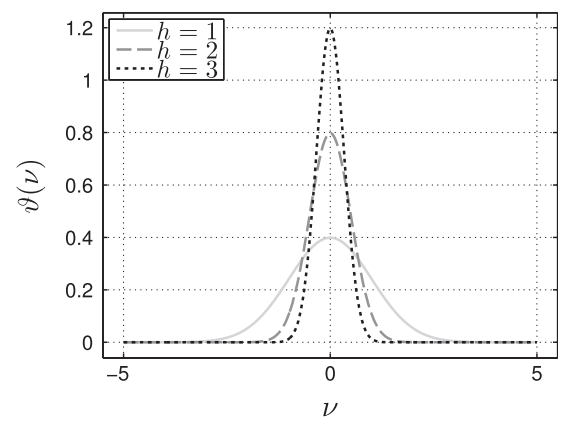

Gaussian

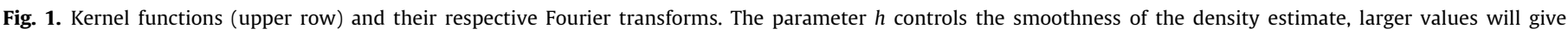
smoother estimates. 
In such case, an approximate function can be used to describe the weights relation to the data, e.g. described as a static function $w(\cdot)$ such that $w_{n}=w\left(y_{k, n}\right)$. The use of such representation of the weights is illustrated in Section 4.2.

\subsubsection{Choosing $\mathbf{w}$ - linear discriminant analysis}

A simple criterion is to maximize the average difference between the means of the datasets $\mathbf{Y}_{K_{0}}^{0}$ and $\mathbf{Y}_{K_{f}}^{f}$. For $\mathbf{Y}_{K_{0}}^{0}$ (and similarly for $\mathbf{Y}_{K_{f}}^{f}$ ), the average of the weighted data is given by

$$
\bar{\mu}^{0} \triangleq \frac{1}{N} \sum_{n=1}^{N}\left[\frac{1}{K_{0}} \sum_{k=1}^{K_{0}} w_{n} y_{k, n}^{0}\right]=\frac{1}{N} \sum_{n=1}^{N} w_{n} \underbrace{\left[\frac{1}{K_{0}} \sum_{k=1}^{K_{0}} y_{k, n}^{0}\right]}_{\triangleq \mu_{n}^{0}}=\frac{1}{N} \mathbf{w}^{T} \boldsymbol{\mu}^{0} .
$$

The average distance $\bar{\mu}^{f}-\bar{\mu}^{0}$ is thus proportional to $\mathbf{w}^{T}\left(\boldsymbol{\mu}^{f}-\boldsymbol{\mu}^{0}\right)$ and the objective is to choose $\mathbf{w}$ which maximizes the expression. This problem is equivalently found in LDA. Constraining $\mathbf{w}$ to unit length $\mathbf{w}^{T} \mathbf{w}=1$ (otherwise the criterion can be made arbitrarily large), it is possible to find that the optimal choice is according to (see e.g. [4, Exercise 4.4]),

$\mathbf{w}^{*} \propto\left(\boldsymbol{\mu}^{f}-\boldsymbol{\mu}^{0}\right)$.

A criterion based only on the distance between the datasets means does not consider the variability found within each dataset, e.g. caused by disturbances and noise. An alternative is to consider maximum average separation while giving small variability within each dataset. The average value of the weighted variance vector over $k$ for $\mathbf{Y}_{K_{0}}^{0}$ (and similarly for $\mathbf{Y}_{K_{f}}^{f}$ ) is given by

$$
\begin{aligned}
\bar{s}^{0} & \triangleq \frac{1}{N} \sum_{n=1}^{N}\left[\frac{1}{K_{0}} \sum_{k=1}^{K_{0}}\left(w_{n} y_{k, n}-w_{n} \mu_{n}^{0}\right)^{2}\right] \\
& =\frac{1}{N} \sum_{n=1}^{N} w_{n}^{2} \underbrace{\left[\frac{1}{K_{0}} \sum_{k=1}^{K_{0}}\left(y_{k, n}-\mu_{n}^{0}\right)^{2}\right]}_{\triangleq S_{n}^{0}}=\frac{1}{N} \mathbf{w}^{T} S^{0} \mathbf{w},
\end{aligned}
$$

where $S^{0}$ is a diagonal matrix with diagonal elements given by $S_{n}^{0}$. Defining the total within class variation as $\bar{s}^{f}+\bar{s}^{0}$, the following criterion can be used

$$
\frac{\left(\bar{\mu}^{f}-\bar{\mu}^{0}\right)^{2}}{\bar{s}^{f}+\bar{s}^{0}} \propto \frac{\mathbf{w}^{T}\left(\boldsymbol{\mu}^{f}-\boldsymbol{\mu}^{0}\right)\left(\boldsymbol{\mu}^{f}-\boldsymbol{\mu}^{0}\right)^{T} \mathbf{w}}{\mathbf{w}^{T}\left(S^{f}+S^{0}\right) \mathbf{w}},
$$

which is a special case of the Fisher criterion in LDA. It can be shown, see e.g. [4], that solutions for this problem satisfy

$\mathbf{w}^{*} \propto\left(S^{f}+S^{0}\right)^{-1}\left(\boldsymbol{\mu}^{f}-\boldsymbol{\mu}^{0}\right)$.

That is, each weight $w_{n}^{*}$ is proportional to the ratio between the average changes caused by faults, $\mu_{n}^{f}-\mu_{n}^{0}$, and the total variability found in the data, $s_{n}^{f}+s_{n}^{0}$, caused by disturbances and noise.

\section{Wear monitoring in an industrial robot joint}

In this first application, the objective is to determine the presence of excessive levels of wear, $w$, in the gearbox of a robot joint. Because increased wear levels may lead to increased friction in the gearbox, it is possible to monitor friction to infer about wear. Since the friction torques must be overcome by the applied motor torques, $\tau$, during its operation, it is possible to extract information about friction from available data. Friction is however not only affected by wear, but also by unknown disturbances, such as variations of load torques, $\tau_{l}$, and the lubricant temperature, $T$, see Fig. 2. ${ }^{2}$ While it may be simpler to ensure constant load conditions,

\footnotetext{
2 Throughout the paper, all torque quantities are normalized to the maximum allowed torque and are therefore dimensionless.
}

temperature is the result of complicated losses mechanisms in the joint and heat exchanges with the environment which are difficult to control. The effects of $\tau_{l}$ and $T$ to friction are in fact comparable to those caused by w (recall Fig. 2) and the problem is therefore challenging. In [5], a model-based approach was suggested for this problem based on the identification of a wear parameter from friction data collected under dedicated experiments. A shortcoming with this approach lies in the need of a detailed friction model, which requires a large amount of experiments to be found. The data-driven approach suggested here is considered as an alternative which requires little design effort since no model development is needed.

Since $\tau$ is affected by friction, and thus by wear, torque (current $^{3}$ ) data are considered for the generation of fault indicators. The monitored data are collected from repetitive executions of a trajectory $\mho$. Relating to the notation introduced in Section 2, the deterministic unknown input of interest, $\mathbf{f}$, is the wear level $w$ and the monitored data, $\mathbf{y}$, is $\tau$ which is affected by disturbances, $\mathbf{d}$, caused by load $\tau_{l}$ and temperature $T$ and by measurement noise $\mathbf{v}$. A trajectory, $v$, is a known deterministic sequence used as a reference to the motion control, i.e. it relates to $\mathbf{r}$. In many applications, the same trajectory is executed over and over again, ensuring a repetitive behavior of the robot. For the results presented here, data collected from the execution of a trajectory $\mho$ based on a test-cycle are used. Torque data collected form this trajectory can be seen in Fig. 3(a). Nominal torque data, $\tau^{0}$, are achieved from the execution of $\mho$ when the gearbox is new and no significant wear is present.

Section 4.1 presents experimental results for the wear monitoring problem when the changes in disturbances are kept small. In Section 4.2, temperature disturbances are introduced in simulation studies and the use of weights described in Section 3.3 are used to illustrate how to reduce sensitivity to disturbances.

\subsection{Experimental wear monitoring under constant disturbances}

Accelerated wear tests were performed with ABB IRB 6620 industrial robots with the objective of studying the wear effects. In an accelerated wear test, the robot is run under high load and stress levels for several months or years until the wear levels become significant and maintenance is required. Throughout the tests, the trajectory $\mho$ was executed regularly a total of $K$ times yielding a dataset $\left[\tau_{0}, \ldots, \tau^{K-1}\right]$. The data were collected from axis two of the robot which is equipped with a rotary vector gearbox type. The experiments were performed in a lab, in a setup to avoid temperature variations ${ }^{4}$ and no load variations were present. It is thus considered that the disturbances had a repetitive behavior $\mathbf{d}_{k}=\mathbf{d}$ over all batches. The data batch taken from the start of the operation of a gearbox is considered to be fault-free and is labeled as nominal, $\tau^{0}$. The quantities $\operatorname{KL}\left(\hat{p}^{0} \| \hat{p}_{k}\right)$ are computed for $k=1, \ldots, K-1$ and are used as fault indicators. Data collected from two accelerated wear tests are considered here. For an illustration of the wear behavior during the experiments, the friction levels in the joint were estimated using a dedicated experiment (see [6] for a description of such experiment) at each $k$ th execution of $v$ and are shown as function of motor speed $\dot{\varphi}$.

For the first case, displayed in Fig. 3, $K=36$ batches of data are considered. From analyses of the friction levels in Fig. 3(c), it is possible to note that wear only starts to considerably affect friction after $k=25$. The effects of wear to the torque sequences, shown

\footnotetext{
${ }^{3}$ In the application, a torque estimate based on a constant relationship between current measurements is used. This simplification is commonly used for control purposes since the current controller has much faster dynamics compared to the dynamics of the robot arm and the such estimate is therefore perceived as the control input signal.

4 The environment temperature was controlled and the experiments were only performed after the robot temperature was expected to be in equilibrium with the environment.
} 


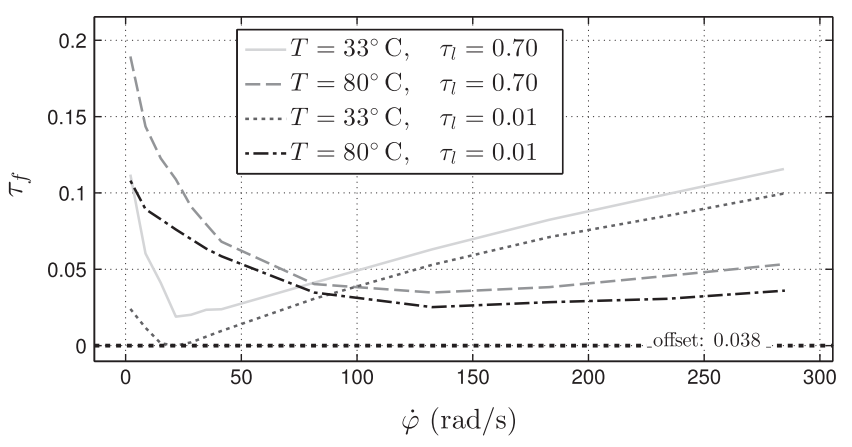

(a) Effects of load and temperature

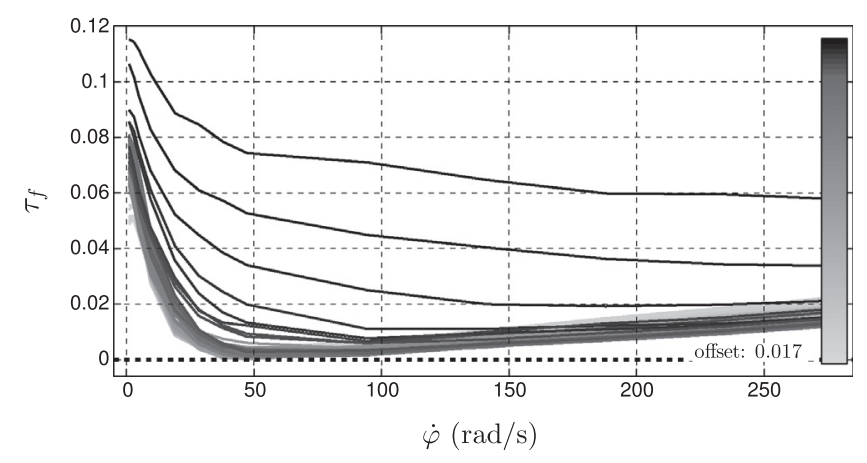

(b) Effects of wear

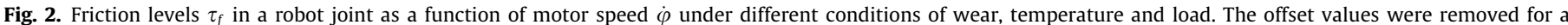

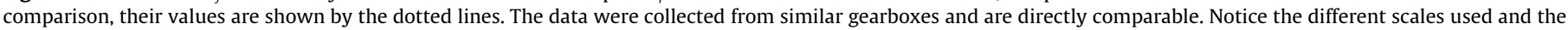

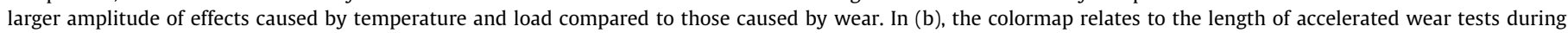
which the curves were registered.

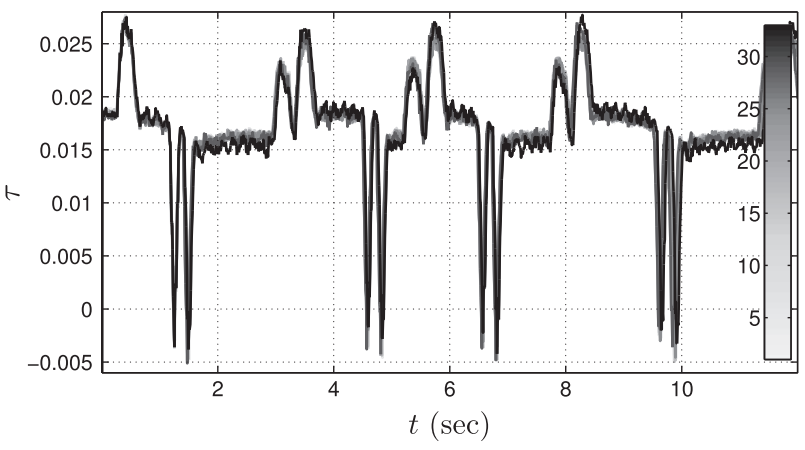

(a) Monitored torque data

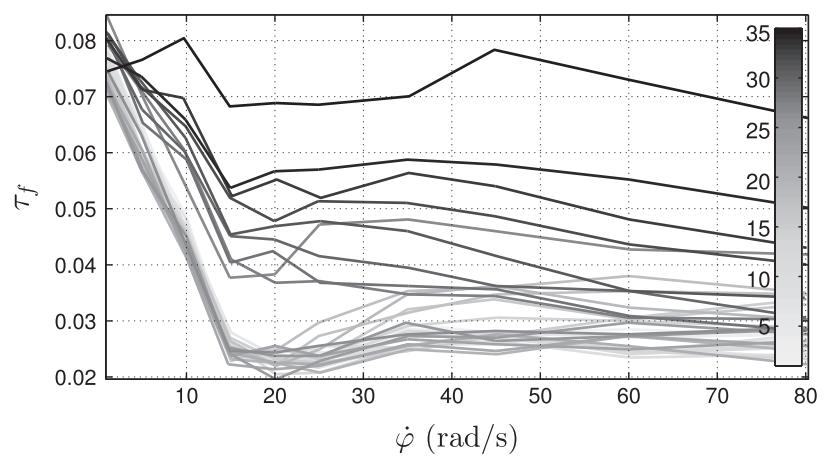

(c) Friction curves

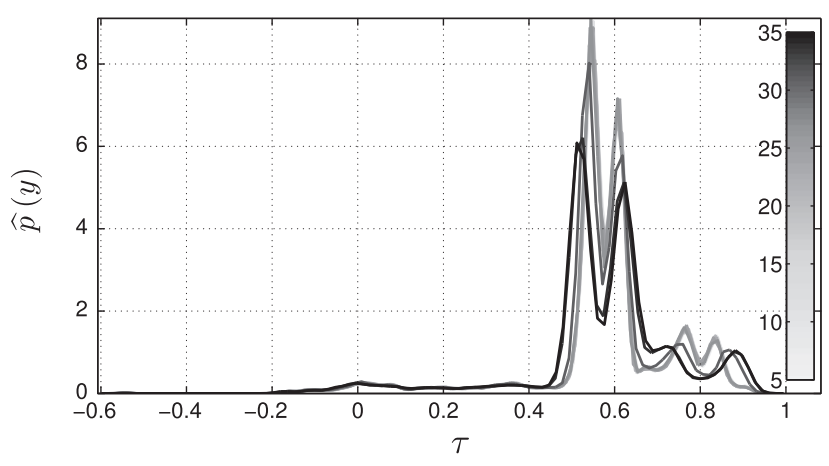

(b) Estimated distributions

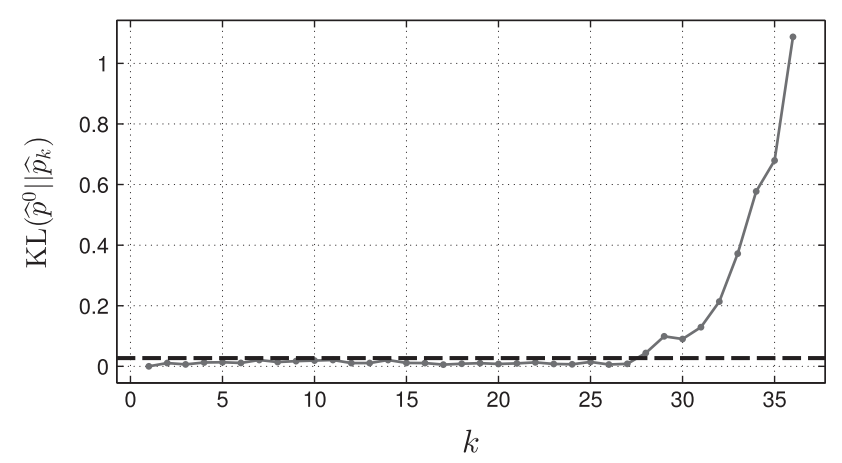

(d) Fault indicator, $\mathrm{KL}\left(\widehat{p}^{0} \| \widehat{p_{k}}\right)$

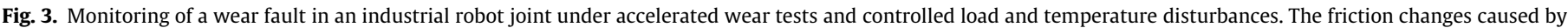

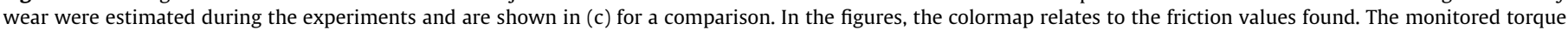

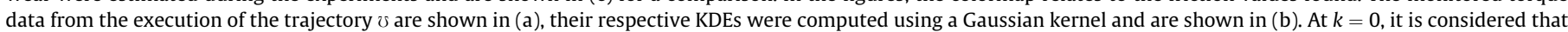

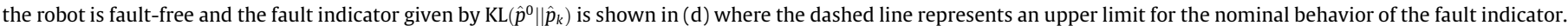
Notice the clear response of the fault indicator to the wear changes.

in Fig. 3(a), appear as small variations in amplitude due to increased friction. The variations in the torque sequences are more easily distinguishable in the distribution domain as seen in Fig. 3(b). Wear affects the location and size of the distribution peaks. Notice further that the distributions are similar for $k \leq 25$ when the robot condition has not significantly changed. The resulting fault indicator, shown in Fig. 3(d), shows a clear response to the changes in friction, remaining close to 0 for $k \leq 25$ and increasing thereafter. To allow for CBM, it is considered that, in this test, a fault should be detected before $k=30$. Using data for $k \leq 25$, the mean and standard deviation for the (considered) nominal behavior of the fault indicator are estimated as $\left[\mu_{0}, \sigma_{0}\right]=\left[1.1910^{-2}, 5.0910^{-3}\right]$. The dashed line in Fig. $3(\mathrm{~d})$ shows the value of $\hbar=\mu_{0}+3 \sigma_{0}$.

The second case, shown in Fig. 4, illustrates the situation where a gearbox is replaced after a wear related failure takes place. A total of $K=111$ data batches are collected during accelerated wear tests using the same test-cycle. A gearbox failure occurs at $k=73$ when it is replaced by a new one. The friction curves related to the faulty gearbox are shown in Fig. 4(c), where it can be noticed that the 
changes due to wear start to appear around $k=64$. The related distribution estimates for this gearbox are shown in Fig. 4(a) where a similar behavior as in the previous case can be noticed, with changes in the size and position of the distributions' peaks. The data densities for the replaced gearbox can be seen in Fig. 4(b) where it is possible to notice that no significant variations are present. The fault indicator is shown in Fig. 4(d), where, as in the previous case, $\hbar=\mu_{0}+3 \sigma_{0}$. The filled circle highlights the moment when the gearbox was replaced. As it can be seen in these studies, an early detection of the increased wear is made possible with the use of the proposed fault indicator, allowing for CBM.

\subsection{Simulated wear monitoring under temperature disturbances}

Simulation studies were carried out to illustrate the ideas to reduce sensitivity to disturbances and noise presented in Section 3.3. The use of simulations allow for more detailed studies of the effects of the disturbances compared to what could be achieved based on experiments in a feasible manner. The simulation model is based on the two link manipulator with elastic gear transmission presented in the benchmark problem of [31]. With the objective of studying friction changes related to wear in a robot joint, the static friction model described in [5] is included in the simulation model. The friction model included was developed from empirical studies in a robot joint and describes the effects of angular speed $\dot{\varphi}$, manipulated load torque $\tau_{l}$, temperature $T$, and wear w.

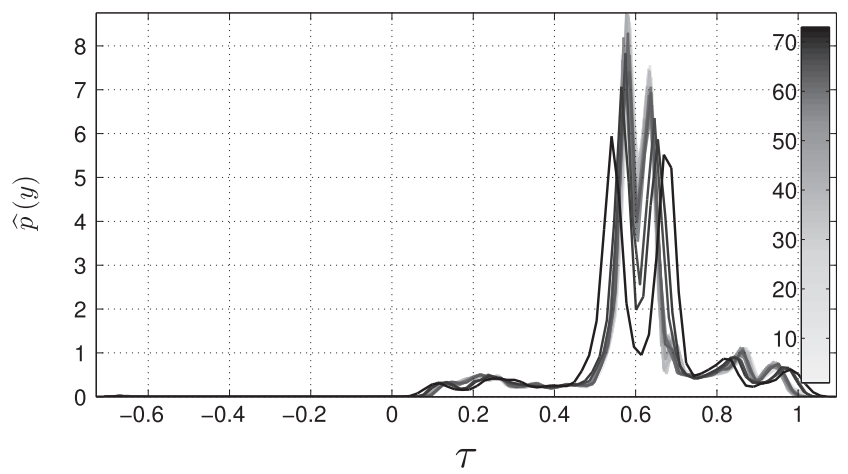

(a) Estimated distributions for $0 \leq k \leq 72$

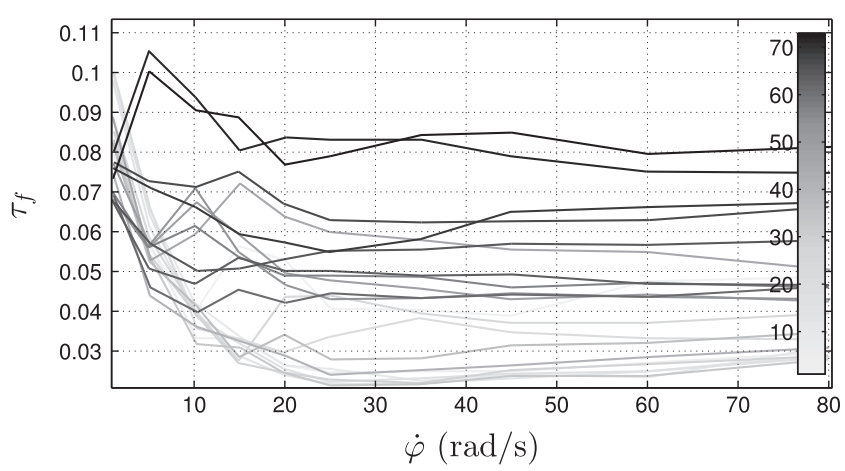

(c) Friction curves for $0 \leq k \leq 72$

\subsubsection{Finding the weights $\mathbf{w}$}

According to the procedures described in Section 3.3, a labeled dataset is needed in order to find the optimal weights. The dataset is achieved here based on simulations of the same test-cycle trajectory $\mho$ used in Section 4.1. Each labeled dataset $\mathbf{Y}_{K_{0}}^{0}$ and $\mathbf{Y}_{K_{f}}^{f}$ contain $K_{0}=K_{f}=100$ batches with torque data generated from the sets

$\mathcal{Y}_{r}^{0}=\left\{\tau_{k}: \mathrm{w}_{k}=0, T_{k} \sim U\left[\underline{T}, \underline{T}+\Delta_{T}\right], \mho_{k}=\mho\right\}$

$\mathcal{Y}_{r}^{f}=\left\{\tau_{k}: \mathrm{w}_{k}=\mathrm{w}_{c}, T_{k} \sim U\left[\underline{T}, \underline{T}+\Delta_{T}\right], \mho_{k}=\mho\right\}$

respectively, where $w_{c}=35$ is a wear level considered critical to generate an alarm (see [5] for details of the wear model). Here, $T$ is considered random, with uniform distribution given by $\underline{T}=30^{\circ} \mathrm{C}$ and $\Delta_{T}=40^{\circ} \mathrm{C}$. This assumption is carried out for analysis purposes and allows for great variations of temperature disturbances.

The optimal weights given in (14) and (15) depend on the average changes found in the data, $\mu_{n}^{f}-\mu_{n}^{0}$, and the total variability, $s_{n}^{f}+s_{n}^{0}$. These quantities are computed based on the labeled dataset and are displayed in Fig. 5(a) as a function of the motor speed $\dot{\varphi}$. As it can be seen, the optimal weights present a strong correlation with $\dot{\varphi}$. This is not a surprise since the effects of $w$ and $T$ depend on $\dot{\varphi}$, recall Fig. 2 . In the same figure, worst case estimates along speed are also shown (solid lines), i.e. $\mu_{n}^{f}-\mu_{n}^{0}$ closest to zero and largest $s_{n}^{f}+s_{n}^{0}$. Fig. 5(b) presents the ratio for such worst case estimates, which are considered as the optimal weights according to (15). The solid line in Fig. 5(b) is a function approximation of the optimal weights given by

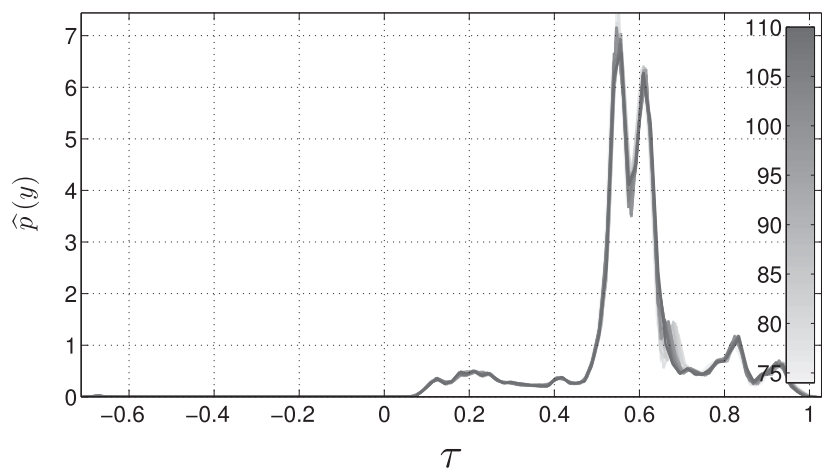

(b) Estimated distributions for $73 \leq k \leq 110$

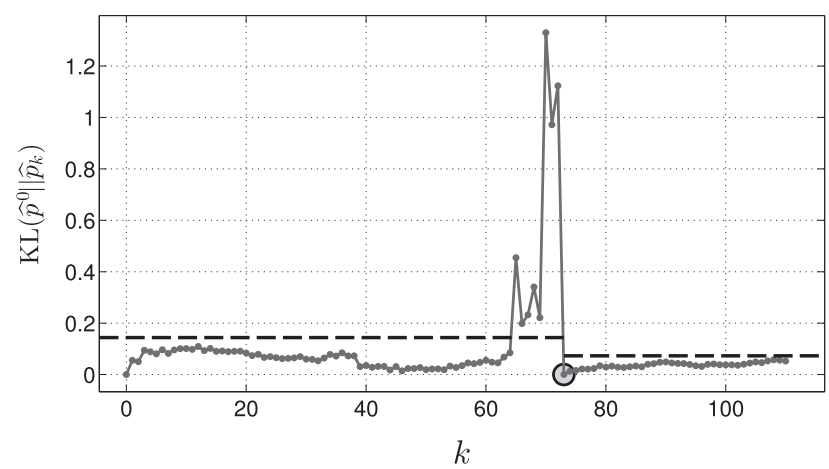

(d) Fault indicator, $\mathrm{KL}\left(\widehat{p}^{0} \| \widehat{p}_{k}\right)$

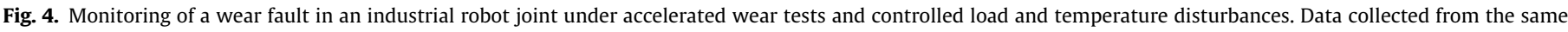

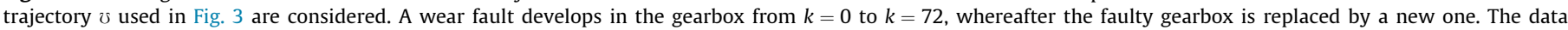

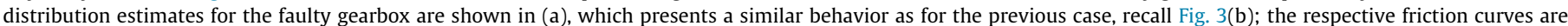

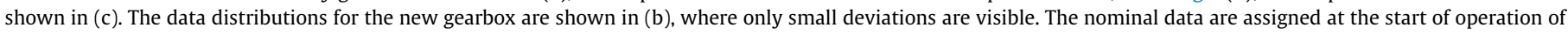

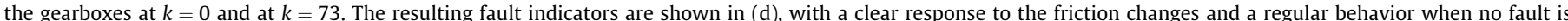

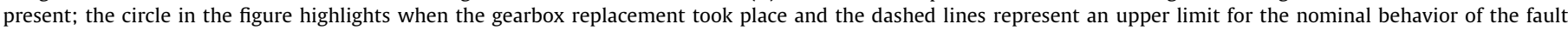
indicator. 


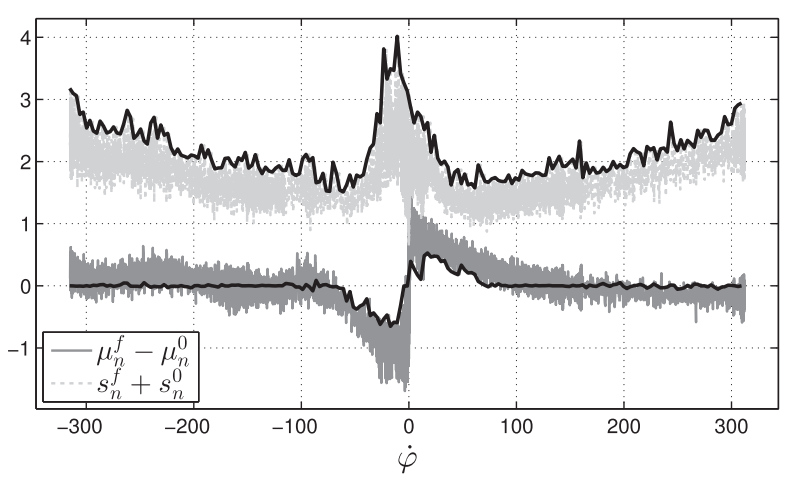

(a) Average effects

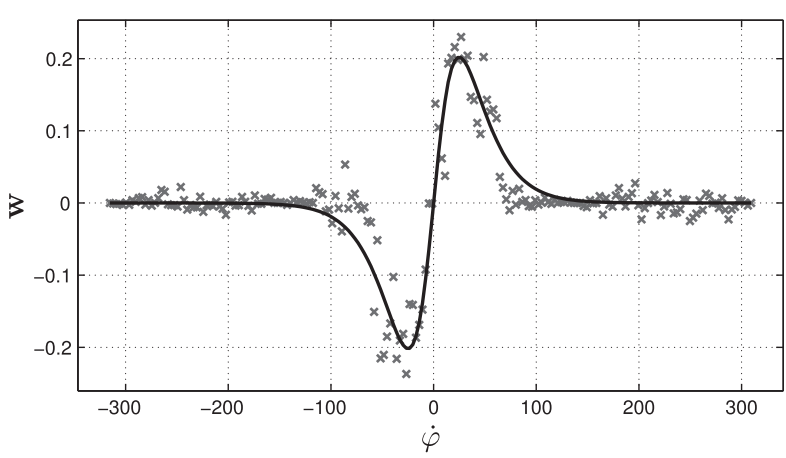

(b) Optimal weights

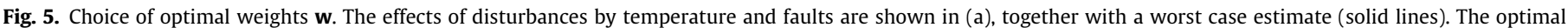

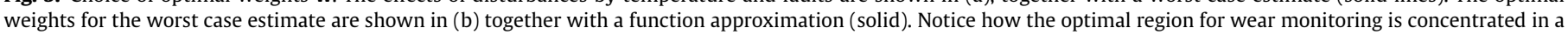
narrow speed range.

$w(\dot{\varphi})=\operatorname{sech}(\beta \dot{\varphi}) \tanh (\alpha \dot{\varphi})$

with $\alpha=1.4510^{-2}$ and $\beta=4.5510^{-2}$. The parametrization of the weight vector as a function of $\dot{\varphi}$ allows for a more general use of the optimal weights and the same weighting function can be used for other trajectories. Effectively, the optimal weighting function selects a speed region that is more relevant for wear monitoring, giving more emphasis to data in low to intermediate speed regions. A similar behavior was also found in [5] for the achievable quality of a wear estimate for different speeds under temperature disturbances.

\subsubsection{Improvements in detection performance}

To illustrate the possible improvements achieved with the use of the weighting function, an abrupt change detection is considered. Given a nominal data batch $\tau^{0} \in \mathcal{Y}_{r}^{0}$, the detection problem is to decide whether a test batch $\tau_{k}$ belongs to $\mathcal{Y}_{r}^{0}$ or $\mathcal{Y}_{r}^{f}$ based on the fault indicator $\operatorname{KL}\left(\hat{p}^{0} \| \hat{p}_{k}\right)$ and where the sets $\mathcal{Y}_{r}^{0}$ and $\mathcal{Y}_{r}^{f}$ are given by (16). This corresponds to the following hypotheses

$\mathcal{H}^{0}: \tau_{k} \in \mathcal{Y}_{r}^{0}, \quad \mathcal{H}^{1}: \tau_{k} \in \mathcal{Y}_{r}^{f}$

where $\mathcal{H}^{0}$ indicates that no wear fault is present, with $\mathrm{w}=0$, and $\mathcal{H}^{1}$ indicates presence of a wear fault of size $\mathrm{w}_{c}$. For given values of $\mathrm{w}_{c}, T$ and $\Delta_{T}$, the test-cycle trajectory $\mho$ is simulated to generate data according to (16). The probability densities of the fault indicator under each hypothesis is estimated based on 5000 Monte Carlo runs with and without the use of the weighting function. Based on the hypotheses densities', the probability of detection $P_{d}$ is computed according to (4) for a threshold check when the probability of false alarm is $P_{f}=0.01$.

Fig. 6(a) presents the achieved $P_{d}$ as a function of $\Delta_{T}$ for the fixed $w_{c}=35$ and $\underline{T}=30^{\circ} \mathrm{C}$ with and without the use of the weighting function. Notice that the use of the weighting function considerably improves $P_{d}$ under temperature variations, but for too large $\Delta_{T}$ it becomes difficult to distinguish the effects. A similar study is performed to illustrate how $\mathrm{w}_{c}$ affects the performance. Fig. 6(b) presents $P_{d}$ as a function of $\mathrm{w}_{c}$ for the fixed $\Delta_{T}=25^{\circ} \mathrm{C}$ and $\underline{T}=30^{\circ} \mathrm{C}$. The improvements achieved using the weighted data are clear.

\section{Gearbox monitoring based on vibration data}

In this application, vibration data collected from the gearbox test rig described in $[19,23]$ are considered. The test rig is composed of a motor coupled to a gearbox with three shafts and four spur gears. It is possible to study the effects of different types of gear faults in the rig by replacing healthy gears with damaged ones. Four different fault types (modes) are considered:
- $m=0$ : healthy gears are used,

- $m=1$ : a gear at the input shaft is damaged,

- $m=2$ : a gear at the output shaft is damaged,

- $m=3$ : a gear at the input and a gear at the output shafts are damaged.

Fault detection approaches for this problem have been proposed in [19] with the use of Hilbert and wavelet packet transform and in [23] with a combination of Wavelet transform and time domain averaging. In these approaches, the data are transformed to a time-frequency domain where different faults can be distinguished based on inspection of the transformed data. The distribution domain approach proposed here is considered as an alternative for fault diagnosis.

Data collected from an accelerometer placed close to the output shaft are considered for the analyses. All data collection is performed under constant load and speed conditions in all settings, ensuring a repetitive behavior. For each different condition, $8 \times 1024$ samples are available with data sampled at $2.56 \mathrm{KHz}$. The datasets are divided in $K=8$ batches with $N=1024$ samples to form $\mathbf{Y}_{K}^{m}$ for each mode $m$. Fig. 7 (a) shows the fist data batch $\mathbf{y}_{1}^{m}$ for each mode $m$, notice that it is difficult to distinguish differences in the data sequences. In Fig. 7(b), the density estimates $\hat{p}_{k}^{m}(y)$ for every batch in every mode are displayed. Notice the smaller variability of the distribution estimates within each mode compared to the variability found between modes.

To evaluate the fault indicator performance to change detection, the distance $\operatorname{KL}\left(\hat{p}^{0} \| \hat{p}_{k}\right)$ is computed for every possible pair such that $\mathbf{y}^{0} \in \mathbf{Y}_{K}^{0}$ and $\mathbf{y}_{k} \in \mathbf{Y}_{K}^{m}$ for $m \in[0,1,2,3]$. Since the distances are computed in pairs, this gives 28 samples from the nominal set $\mathcal{D}^{0,0}\left(K=8\right.$ combined two by two) and $K^{2}=64$ samples from the sets $\mathcal{D}^{0, m}$ for $m \in[1,2,3]$. The hypotheses densities $p\left(\mathrm{KL} \mid \mathcal{H}^{m}\right)$ are estimated based on these samples and are shown in Fig. 7(c). Notice the clear separation between the null hypothesis density, $p\left(\mathrm{KL} \mid \mathcal{H}^{0}\right)$, from the alternatives. For $P_{f}=0.01, P_{d}$ is computed based in (4) for a threshold check when deciding between $\mathcal{H}^{0}$ and $\mathcal{H}^{m}$ for $m \in[1,2,3]$; the achieved values are $P_{d}=[1,0.991,1]$. To illustrate the approach to isolation as given in (5), the first data batch in each mode, $\mathbf{y}_{1}^{m}$, is considered available. The isolation criterion given by $m^{*}=\arg \min _{m} \operatorname{KL}\left(\hat{p}_{1}^{m}|| \hat{p}_{k}\right)$ chooses the correct mode for all $(K-1) \times 4$ remaining data batches.

\section{Conclusions and future work}

The suggested framework considers the monitoring of changes in the distribution of data batches. Because no prior knowledge is assumed about the data behavior, nonparametric kernel density 


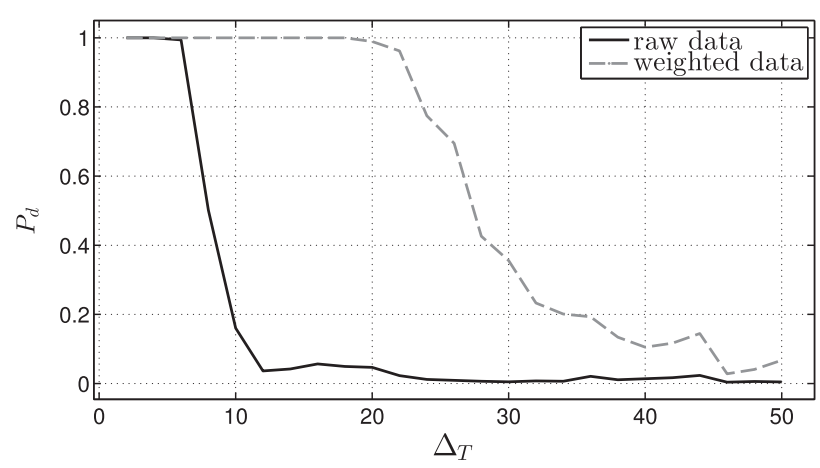

(a) Temperature variations

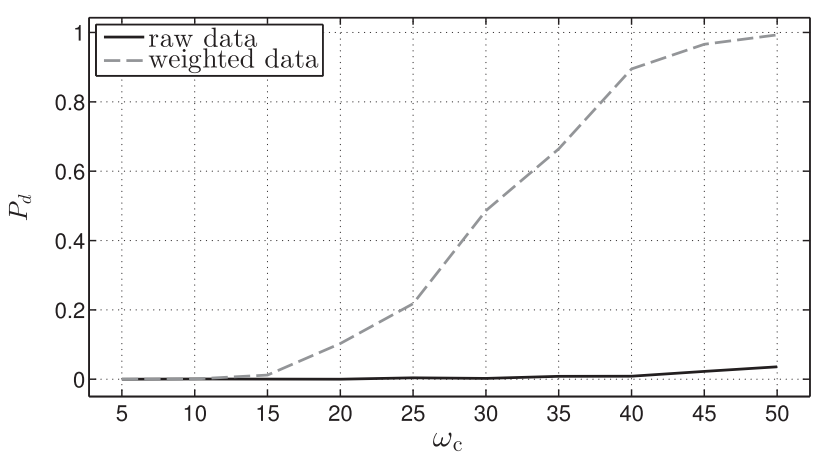

(b) Fault size

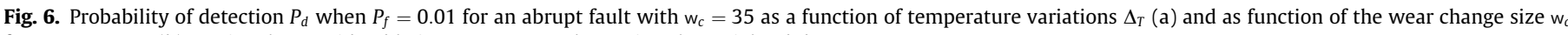
form $\Delta_{T}=25{ }^{\circ} \mathrm{C}$ (b). Notice the considerable improvements when using the weighted data.

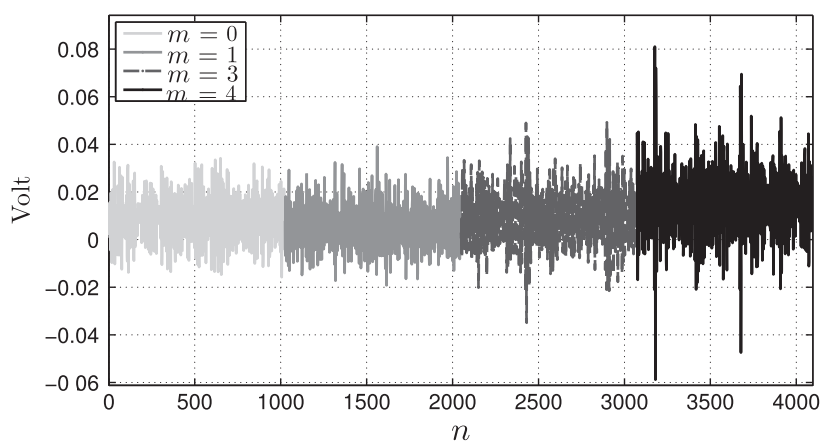

(a) Data sequences

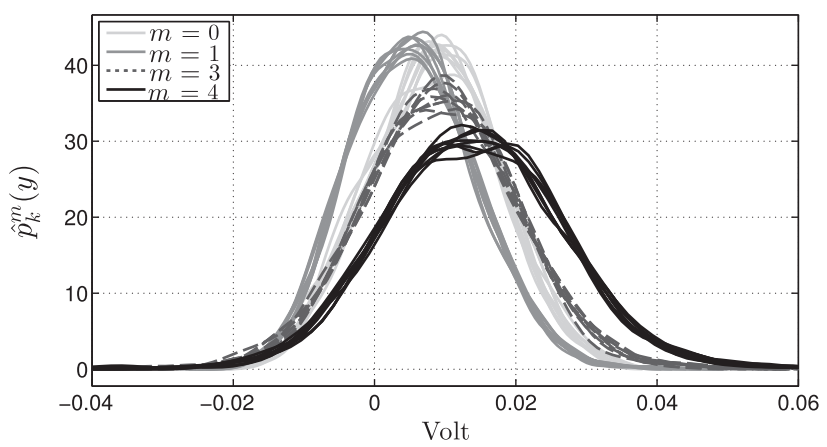

(b) Density estimates

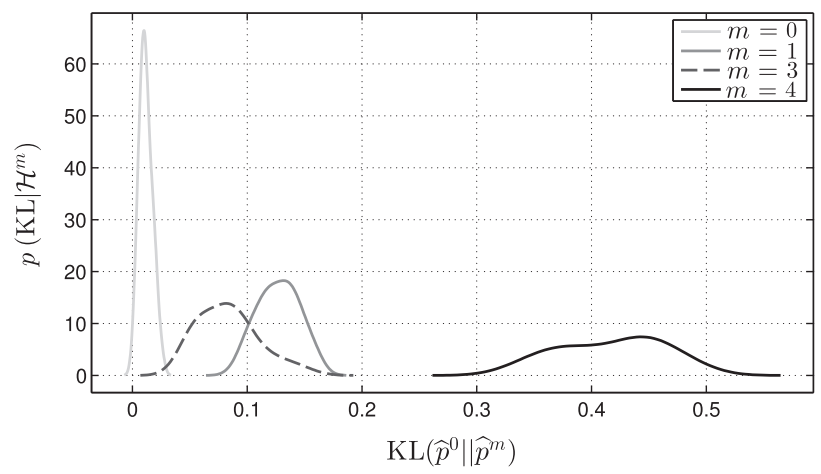

(c) Fault indicator behavior

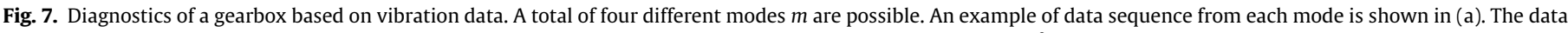

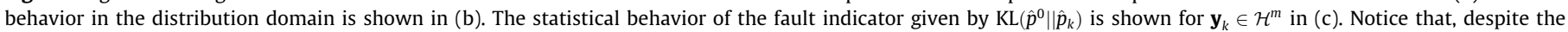
densities in (b) being similar, the fault indicator clearly indicates the presence of a change in (c).

estimates are used, which give great flexibility, are simple to implement and have an inherent smoothing behavior. The validity of the framework and methods were illustrated with promising results on real case studies and simulations for gearbox monitoring in robotics and rotating machines. An important advantage of the framework presented is that no model or expert knowledge of the system are required. Furthermore, it gives an alternative for systems where faults affect the data amplitude but where stationary, periodic or linear behaviors are difficult or not possible, as in the robotics application presented.

As future work, it would be interesting to further investigate the methods applicability to other domains and faults. In this direction, the derivation of conditions for diagnosability are relevant. The determination of a change in data collected under repetitive conditions simplifies the diagnostics problem considerably. In general however, it might not possible to ensure the same repetitive behavior of the system. This is the case, for example, in the industrial robotics application where trajectories are normally only repeated trough a certain period, depending on the manufacturing plan. Thus, it would relevant to study approaches to handle systems with a varying repetitive behavior. The effects of different kernel functions for the KDE, choice of bandwidth parameter and use of different distances between densities are also important. 


\section{Acknowledgments}

This work was supported by $\mathrm{ABB}$ and the Vinnova Industry Excellence Center LINK-SIC at Linköping University. The authors would also like to thank Professor Sirish L. Shah from the University of Alberta, Canada, for sharing the data used in the studies of Section 5.

\section{References}

[1] Aguilar-Lopez R, Martinez-Guerra R. Robust state estimation for repetitive operating mode process: application to sequencing batch reactors. Chem Eng J 2007;126(2):155-61.

[2] Alvarado IA, Findeisen R, Kuhl P, Allgower F, Limón D. State estimation for repetitive processes using iteratively improving moving horizon observers. In: CDC-ECC'05. 44th IEEE conference on decision and control, 2005 and 2005 European control conference. IEEE; 2005. p. 7756-61.

[3] Basseville M. Distance measures for signal processing and pattern recognition. Signal Process 1989;18(4):349-69.

[4] Bishop CM. Pattern recognition and machine learning. 1st ed. New York (USA): Springer; 2007.

[5] Bittencourt AC, Axelsson P. Modeling and experiment design for identification of wear in a robot joint under load and temperature uncertainties based on friction data. IEEE/ASME Trans Mech 2013;PP(99):1-13.

[6] Bittencourt AC, Gunnarsson S. Static friction in a robot joint-modeling and identification of load and temperature effects. J Dynam Syst Meas Control 2012;134(5).

[7] Bittencourt AC, Saarinen K, Sander-Tavallaey S. A data-driven method for monitoring systems that operate repetitively - applications to wear monitoring in an industrial robot joint. In: Proc of the 8th IFAC SAFEPROCESS, vol. 8. Mexico City, Mexico; 2012.

[8] Bowman AW, Azzalini A. Applied smoothing techniques for data analysis: the Kernel approach with S-plus illustrations (Oxford statistical science series). USA: Oxford University Press; 1997.

[9] Brambilla D, Capisani L, Ferrara A, Pisu P. Fault detection for robot manipulators via second-order sliding modes. IEEE Trans Ind Electron 2008;55(11):3954-63.

[10] Caccavale F, Cilibrizzi P, Pierri F, Villani L. Actuators fault diagnosis for robo manipulators with uncertain model. Control Eng Pract 2009:17(1):146-57.

[11] Cacoullo T. Estimation of a multivariate density. Ann Inst Stat Math 1966;18:179-89.

[12] De Luca A, Mattone R. Actuator failure detection and isolation using generalized momenta. In: Proc of the 2003 IEEE international conference on robotics and automation (ICRA), vol. 1. Taipei, Taiwan; 2003. p. 634-9.

[13] de Silva CW. Vibration monitoring, testing and instrumentation. CRC Press; 2007.

[14] Ding SX, Yin S, Wang Y, Wang Y, Yang Y, Ni B. Data-driven design of observers and its applications. In: Proceedings of the 18th IFAC world congress; 2011.

[15] Dixon WE, Walker ID, Dawson DM, Hartranft JP. Fault detection for robot manipulators with parametric uncertainty: a prediction-error-based approach. IEEE Trans Robot Autom 2000;16(6):3628-34.

[16] Dong J, Verhaegen M, Gustafsson F. Robust fault detection with statistical uncertainty in identified parameters. IEEE Trans Signal Process 2012;60(10):5064-76.

[17] Durrett R. Probability: theory and examples. Cambridge University Press; 2010

[18] Eski I, Erkaya S, Savas S, Yildirim S. Fault detection on robot manipulators using artificial neural networks. Robot Comput-Int Manuf 2011;27(1):115-23.
[19] Fan X, Zuo MJ. Gearbox fault detection using hilbert and wavelet packet transform. Mech Syst Signal Process 2006;20(4):966-82.

[20] Filaretov V, Vukobratovic M, Zhirabok A. Observer-based fault diagnosis in manipulation robots. Mechatronics 1999;9(8):929-39.

[21] Freyermuth B. An approach to model based fault diagnosis of industrial robots. In: Proc of the 1991 IEEE international conference on robotics and automation (ICRA), vol. 2. Sacramento, USA; 1991. p. 1350-6.

[22] Guo SC, Yang MH, Xing ZR, Li Y, Qiu JQ. Actuator fault detection and isolation for robot manipulators with the adaptive observer. Adv Mater Res 2012;482484(8):529-32.

[23] Halim EB, Choudhury MS, Shah SL, Zuo MJ. Time domain averaging across all scales: a novel method for detection of gearbox faults. Mech Syst Signal Process 2008;22(2):261-78.

[24] Isermann R. Fault-diagnosis systems - an introduction from fault detection to fault tolerance. Springer; 2006.

[25] Isermann R. Fault-diagnosis applications - model-based condition monitoring: actuators, drives, machinery, plants, sensors, and fault-tolerant systems. New York: Springer; 2011.

[26] Jones M, Henderson D. Maximum likelihood kernel density estimation: on the potential of convolution sieves. Comput Stat Data Anal 2009;53(10):3726-33.

[27] Li X, Zhou K. A time domain approach to robust fault detection of linear timevarying systems. Automatica 2009;45(1):94-102.

[28] Ljung L. System identification: theory for the user. 2nd ed. Prentice Hall PTR; 1998.

[29] Marton L, van der Linden F. Temperature dependent friction estimation: application to lubricant health monitoring. Mechatronics 2012;22(8):1078-84.

[30] McIntyre M, Dixon W, Dawson D, Walker I. Fault identification for robot manipulators. IEEE Trans Robot 2005;21(5):1028-34.

[31] Moberg S, Öhr J, Gunnarsson S. A benchmark problem for robust control of a multivariable nonlinear flexible manipulator. In: Proc of the 17th IFAC world congress; 2008.

[32] Olsson E, Funk P, Xiong N. Fault diagnosis in industry using sensor readings and case-based reasoning. J Intell Fuzzy Syst 2004;15:10.

[33] Parzen E. On estimation of a probability density function and mode. Ann Math Stat 1962;33(3):1065-76.

[34] Reid MD, Williamson RC. Information, divergence and risk for binary experiments. J Mach Learn Res 2011;12:731-817.

[35] Rogers E, Galkowski K, Owens DH. Control systems theory and applications for linear repetitive processes, vol. 349. Springer; 2007.

[36] Rogers E, Galkowski K, Owens DH. Two decades of research on linear repetitive processes. Part i: Theory. In: Proceedings of the 8th international workshop on multidimensional systems (nDS), 2013; 2013. p. 1-6.

[37] Rogers E, Galkowski K, Paszke W, Owens DH. Two decades of research on linear repetitive processes. Part ii: Applications. In: Proceedings of the 8th international workshop on multidimensional systems (nDS), 2013; 2013. p. 16.

[38] Sander-Tavallaey S, Saarinen K. Backlash identification in transmission unit. In: Proc of the 2009 IEEE control applications \& intelligent control; 2009. p. 1325-31.

[39] Sulikowski B, Gałkowski K, Rogers E, Owens DH. Output feedback control of discrete linear repetitive processes. Automatica 2004;40(12):2167-73.

[40] Taylor JI. The vibration analysis handbook. Vibration consultants; 1994

[41] Van Trees HL, Bell KL. Detection, estimation and modulation theory. Part I. 2nd ed. New York: Wiley; 2013.

[42] Wang Y, Ma G, Ding SX, Li C. Subspace aided data-driven design of robust fault detection and isolation systems. Automatica 2011;47(11):2474-80.

[43] Wu L, Su X, Shi P. Mixed $H_{2} / H_{\infty}$ approach to fault detection of discrete linear repetitive processes. J Frank Inst 2011;348(2):393-414.

[44] Yin S, Ding SX, Abandan Sari AH, Hao H. Data-driven monitoring for stochastic systems and its application on batch process. Int J Syst Sci 2013;44(7):1366-76. 